\title{
Accidental encounters: the chance to solve a mystery
}

\section{Masatoshi Takeichi}

Cells organize into tissues by adhering to one another. Such intercellular associations can be disrupted artificially and, under the right culture conditions, the dissociated cells can re-aggregate and reconstitute their original tissue-like structures, as demonstrated by early pioneering studies. When I entered graduate school, the molecular mechanisms governing these striking cellular behaviours, including the formation of the initial cell-cell contacts, were largely unresolved.

At the beginning of my career, I was interested in lens cell differentiation. Lens epithelial cells differentiate into lens fibres, a process that was thought to depend on unidentified factors released from the retina. I set out to characterize these putative factors by culturing retinal cells and collecting the culture medium 'conditioned' by these cells, thinking that it might contain the factors I sought. But when I grew lens cells in this conditioned medium, nothing seemed to happen. After much fruitless effort, I finally noted a difference; lens cells suspended in the conditioned medium attached to the culture dish more slowly than lens cells in the control medium. This unexpected effect had nothing to do with lens differentiation, but attracted my interest nonetheless, for I felt it should be somehow possible to analyse the underlying mechanisms. But the tools needed to take a mechanistic approach to problems in cell differentiation had yet to be developed, and I eventually gave up on the lens.

I was in Tokindo Okada's laboratory at Kyoto University at this time. Although his main interest was in cell differentiation, he encouraged students to learn about morphogenesis as well. He inspired his students

Masatoshi Takeichi is Director of the RIKEN Center for Developmental Biology, 2-2-3 MinatojimaMinamimachi, Chuo-ku, Kobe 650-0047, Japan. e-mail: takeichi@cdb.riken.jp to gain broad insights into developmental mechanisms, and provided us with a learning environment that, I believe, was critical in developing my interest in topics such as cell adhesion, which were not widely popular among developmental biologists.

I continued studying cell adhesion and subsequently found that the mechanisms of cell-cell and cell-substrate adhesion require different divalent cations $\left(\mathrm{Ca}^{2+}\right.$ and $\mathrm{Mg}^{2+}$, respectively) and through this work I became convinced that cells must have multiple adhesion mechanisms. But as the necessary techniques remained unavailable, I still could not test this idea at the molecular level. Around this time, I went to do a postdoctoral fellowship in Richard Pagano's laboratory at the Carnegie Institution of Washington, Department of Embryology, and began to explore the mechanisms behind liposome-cell membrane interactions. Soon after the move, however, I noticed something strange. I generally used trypsin to dissociate cells, which would normally reaggregate when cultured in suspension. But when I used the Carnegie recipe do the same thing, the trypsinized cells never re-aggregated. This surprised and interested me, and I set out to solve the mystery. It turned out that the Carnegie trypsin solution contained EDTA to remove divalent cations, whereas my usual solution did not. I confirmed that the presence or absence of $\mathrm{Ca}^{2+}$ in the trypsin solution was the key to the difference I had observed. This led me to hypothesize that cells have an adhesion mechanism that can be disrupted with trypsin, and that $\mathrm{Ca}^{2+}$ confers a protective effect against trypsin digestion. Since the re-aggregation of the cells equipped with this hypothetical mechanism also required $\mathrm{Ca}^{2+}, \mathrm{I}$ called it the $\mathrm{Ca}^{2+}$-dependent adhesion system. I had a strong feeling that this mechanism must be crucial for cell-cell adhesion in animal cells, as it had previously been suggested that $\mathrm{Ca}^{2+}$ is indispensable for the maintenance of animal tissues, and so I decided to follow up on this finding.

Identifying the molecular mechanism underlying $\mathrm{Ca}^{2+}$-dependent adhesion, however, was not an easy task. One promising approach was the immunological one, which was introduced by Günther Gerisch's group to identify adhesion molecules of the cellular slime moulds. The idea behind this approach was that if I could raise antibodies that are able to block cell-cell adhesion, it would enable me to identify antibody targets, which would presumably be adhesion molecules. I tried injecting rabbits with cells, which I had used in the experiments at Carnegie, but these never led to the production of the blocking antibodies I was after. One day, however, I came across a paper by Rolf Kemler and colleagues reporting that antibodies raised against teratocarcinoma cells blocked the compaction of early mouse embryos. Given the morphological similarity between embryonic compaction and $\mathrm{Ca}^{2+}$ dependent cell aggregation, I suspected the underlying mechanisms might be related as well. Indeed, when I switched to teratocarcinoma lines, I was finally able to obtain blocking antibodies. It was these antibodies that led us to identify the first of a large family of molecules now known as cadherins.

Looking back on my early research, it is clear that the struggle to account for some unexpected finding or other has often brought me to a turning point. As scientists, we need to keep ourselves attuned to the uncommon and to avoid blinkering ourselves with dogma. Admittedly, these days I tend to propose rationally designed experiments to my postdocs and students, but I always strive not to overlook any unexpected results from their experiments, and to emphasize to them the importance of this attitude for the advancement of science. 